\title{
A series of JPR Symposia in Volume 123
}

\author{
Hirokazu Tsukaya
}

Published online: 28 November 2009

(C) The Botanical Society of Japan and Springer 2009

Last year the Journal of Plant Research (JPR) was chosen by the Biomedical and Life Sciences Division of the Special Libraries Association (SLA) as one of the 100 most influential journals in biology and medicine over the last 100 years (Tsukaya 2009). The history of a scientific journal should reflect the history of science. Only when this relation works well is the presence of the journal validated, and journals aim to satisfy this goal. Publication of good original articles as early as possible is one such activity of a journal in pursuing that aim. We have made efforts toward that end at JPR. In addition, to provide an overview of progress in a particular research field and simultaneously to stimulate further studies in the field, we have published special issues, the JPR Symposia.

This year, we will publish a series of JPR Symposia in Volume 123. At the beginning in this issue, No. 1, Vol. 123, we see the first JPR Symposium: "Leaf development and evolution." As mentioned in the preface (Tsukaya 2010), understanding of the mechanisms of leaf development has greatly expanded in the years after model plants were adopted for use in this kind of research. As a result, molecular genetics studies on leaf development have become more and more common now. The number of publications on the topic has increased significantly; and evo-devo studies on leaf development have also been started, based on the accumulation of basic knowledge of

H. Tsukaya $(\bowtie)$

Graduate School of Science, The University of Tokyo,

Science Building \#2, 7-3-1 Hongo, Bunkyo-ku,

Tokyo 113-0033, Japan

e-mail: jpreic@biol.s.u-tokyo.ac.jp

H. Tsukaya

National Institute for Basic Biology,

National Institutes of Natural Sciences, Okazaki, Japan the mechanisms of leaf morphogenesis since adopting model plants for studies of leaf morphogenesis began in the 1990s, nearly two decades ago. Twenty years of studies are enough to prepare for the next leap or breakthrough in the coming decades. In fact, some buds of future studies in this research field are already being seen. To celebrate the progress made over the last two decades and looking toward the coming ones, I asked expert researchers around the world, from Australia, Spain, the UK, the US, and of course Japan, to write reviews of the work in this field. The results are seen in the JPR Symposium "Leaf development and evolution." Again, please see the preface (Tsukaya 2010) for details.

There is not only this JPR Symposium; other JPR Symposia are being planned for 2010. The second one will be "Cytoplasmic inheritance", chosen because the year 2010 is the 100th anniversary of the discovery of cytoplasmic inheritance. Cytoplasmic inheritance is a very important aspect of genetic inheritance in eukaryotes. In recent years, the molecular mechanisms behind cytoplasmic inheritance have been extensively explored. Fortunately, many members of the Botanical Society of Japan (BSJ), by which the JPR is published, have contributed to that progress. To celebrate the centennial, I asked Prof. Tsuneyoshi Kuroiwa to edit a JPR Symposium on this topic, and he soon chose authorities, mainly from among the members of BSJ, to write review articles on the progress of research the field. This JPR Symposium will provide a firm basis for the next 100 years' exploration of the mechanisms of cytoplasmic inheritance.

Plans include a special issue in conjunction with the International Congress on Arabidopsis Research (ICAR) 2010 that will be held in Yokohama, Japan, in June; also the Japan-China-Korea A3 Foresight Program on "Quantifying and predicting terrestrial carbon sinks in East 
Asia"; and there are others in the works. I hope the stimulating JPR Symposia will reflect well the present state of plant science, will accelerate the progress of plant science, and will contribute to the history of plant science.

\section{Hirokazu Tsukaya}

Editor-in-Chief, Journal of Plant Research

\section{References}

Tsukaya H (2009) Journal of Plant Research chosen as one of the top 100 journals in biology and medicine over the last 100 years. $\mathbf{J}$ Plant Res 122:353-354

Tsukaya H (2010) Leaf development and evolution. J Plant Res $123: 3-6$ 